\title{
Crachás: efeitos potenciais na dinâmica de comunidades online
}

\author{
Inês Araújo, Carlos Santos, Luís Pedro \\ \& João Batista
}

\begin{abstract}
Resumo
Os MOOC são muitas vezes motores para o desenvolvimento de comunidades online, mas nem sempre é uma tarefa fácil envolver os participantes em interações significativas entre si. É frequente, neste contexto, o recurso a estratégias de gamificação para motivar os participantes e diminuir as desistências. Num MOOC intitulado "Crachás: como usar?" e destinado a professores e formadores, recorreu-se a uma ferramenta de apoio (endorsement) de crachás entre membros. A tarefa sugerida teve como intuito proporcionar uma experiência com Crachás aos participantes, mas também promover a interação entre estes. Através da leitura das apresentações individuais, cada participante deveria apoiar um dos crachás disponíveis, sendo atribuído o crachá com mais apoios. Esta ação desencadeou um grande número de interações que pretendemos analisar neste artigo. Inicialmente ocorreram interações de um para todos os membros como as publicações no grupo, para depois aumentarem as interações de um para um ou vários, como os comentários e o apoio de crachás, que exigem um esforço mais significativo por parte dos utilizadores. Os valores obtidos indicam que o recurso a crachás desinibiu a participação dos membros e que estes reconheceram a atividade como divertida, no entanto consideramos que são necessários mais estudos que validem esta abordagem.
\end{abstract}

Palavras-chave:

SNA; social network analysis; crachás; comunidade de aprendizagem; gamification. 


\title{
Badges: potential effects on the dynamics of online communities
}

\begin{abstract}
MOOCs often trigger the development of online communities, however it is usually difficult to engage participants in meaningful interactions with each other. Often, in this context, the use of gamification strategies motivates participants and decrease potential withdrawals. In a MOOC entitled "Badges: how to use?" targeted to teachers and trainers, a tool to endorse badges between members was used. The aim of the suggested task was to provide an experience with Badges to the participants, but also to promote the interaction between them Through the reading of the individual presentations each participant should support one of the available badges, being awarded the badge with more support. This action has triggered a large number of interactions that are analyzed in this article. Initially one-to-many interactions like group posts occurred, and then we verified a growing number of one-to-one interactions such as comments and endorsement for badges that required a lot more effort. We found that the use of badges disinhibited the participation of members and that they recognized the activity as fun. However, more studies are needed to validate this approach.
\end{abstract}

Keywords: SNA; social network analysis; badges; learning community; gamification.

\section{Badges : effets potentiels sur la dynamique des communautés en ligne}

Résumé: Les MOOC sont souvent des moteurs de développement de communautés en ligne, mais ce n'est pas facile d'engager les participants dans des interactions significatives les uns avec les autres. Souvent, dans ce contexte, s'essaye l'utilisation de stratégies de gamification pour motiver les participants et diminuer les retraits. Dans un MOOC intitulé «Badges: comment user, adressé à enseignants et formateurs, un outil pour l'approbation des badges entre les membres a été utilisé. La tâche suggérée essayé de fournir une expérience avec des badges aux participants, mais aussi de promouvoir l'interaction entre eux. Grâce à la lecture des présentations individuelles, chaque participant devrait soutenir l'un des badges disponibles, en recevant le badge avec plus de soutien. Cette action a déclenché un grand nombre d'interactions que nous avons l'intention d'analyser dans cet article. Au départ, les interactions individuelles comme les publications de groupe ont eu lieu, puis ont augmenté les interactions individuelles telles que les commentaires et le soutien pour les badges qui nécessitaient beaucoup plus d'efforts. Nous avons constaté que l'utilisation de badges désinhibait la participation des membres et quils reconnaissaient l,activité comme amusante, mais nous considérons que diautres études sont nécessaires pour valider cette approche.

Mots-clés: social network analysis; badges; communauté d’apprentissage; gamification.

\section{Badges: efectos potenciales en la dinámica de comunidades en línea}

Resumen: Los MOOC son a menudo motores para el desarrollo de comunidades en línea, pero no siempre es una tarea fácil involucrar a los participantes en interacciones significativas entre sí. Es frecuente, en este contexto, el recurso a estrategias de gamificación para motivar a los participantes y disminuir las desistencias. En un MOOC titulado "Badges: ¿cómo usar?" destinado a profesores y formadores, se recurrió a una herramienta para endorsement de insignias entre miembros. La tarea sugerida tuvo como objetivo proporcionar una experiencia con bagdes a los participantes, pero también promover la interacción entre éstos. A través de la lectura de las presentaciones individuales cada participante debería apoyar una de las insignias disponibles, siendo asignado la insignia con más apoyos. Esta acción desencadenó un gran número de interacciones que pretendemos analizar en este artículo. Inicialmente se produjeron interacciones de uno para todos los miembros como las publicaciones en el grupo, para luego aumentar las interacciones de uno a uno o varios como los comentarios y el apoyo de botones, que exigen mucho más esfuerzo. Hemos encontrado que el uso de insignias desinibiu la participación de los miembros y que reconocen la actividad como divertida, sin embargo, consideramos que se necesitan más estudios que validen este enfoque.

Palabras clave: social network analysis; badges; comunidad de aprendizaje; gamification. 


\section{Introdução}

A aprendizagem em comunidades online está intimamente relacionada com a perceção que o aluno tem das tarefas que lhe são propostas. Fatores como a utilidade da tarefa e o sentimento de competência para executar o que lhe é pedido são decisivos para manter a motivação em contextos de aprendizagem online (Rebollo-Catalán, García-Pérez, Buzón-García, \& Vega-Caro, 2014).

Também parece ser evidente que "(...) learning communities are built and continue only while the course is open and while the teachers are involved in fostering participation" (De Lima \& Zorrilla, 2017, p. 40). Sem que exista um objetivo concreto de execução, com atividades calendarizadas, muitas comunidades acabam por ficar esquecidas pelos seus membros.

No entanto, é também na fase inicial da criação de uma comunidade online que são realizadas ações que promovem a interação, uma vez que a maioria dos participantes não se conhece pessoalmente e, por isso, há dificuldades naturais de interação entre si. Estas ações têm, normalmente, dois objetivos fundamentais. Por um lado, pretendem dar a conhecer as funcionalidades da plataforma utilizada e, por outro lado, pretendem incentivar a interação entre os próprios participantes. É neste âmbito que os elementos de gamificação são por vezes introduzidos em comunidades online e mesmo em MOOCs (Massive Open Online Course) com o intuito de aumentar a motivação e o envolvimento dos participantes nas atividades previstas (Looyestyn et al., 2017).

Um dos elementos de gamificação frequentemente usado são os crachás, que podem ser definidos como uma entidade digital que "(...) consists of a signifying element (the visual and textual cues of the badge), rewards (the earned badge), and the fulfillment conditions which determine how the badge can be earned" (Hamari, 2017, p. 2). As principais vantagens associadas ao uso de crachás são os seguintes (Gee, 2003; Hamari, 2017):

- A definição de objetivos claros que facilitam a execução das tarefas uma vez que, para poderem receber o Crachá, os membros de uma comunidade necessitam de cumprir tarefas que estejam descritas com clareza;

- O feedback imediato proporcionado pela atribuição dos crachás, ou seja, a sua atribuição reconhece automaticamente a execução dos objetivos previamente definidos;

- O impacto do reconhecimento social adquirido em função da divulgação das conquistas, tipicamente associado a sentimentos de realização e orgulho;

- O exemplo fornecido aos outros membros, o que serve de orientação para que outros tentem alcançar o mesmo crachá.

Face a estas evidências, os crachás são muitas vezes utilizados em comunidades online, mas sem que seja analisado que diferenças efetivamente provocam (Hamari, 
2017), ou seja, é analisada a experiência no seu todo não conseguindo identificar qual o contributo que cada elemento efetivamente provocou nos resultados alcançados em termos de motivação, envolvimento nas atividades e interação social. É neste sentido que se recorreu à técnica de Social Network Analysis (SNA) de uma comunidade criada na plataforma SAPO Campus ${ }^{1}$ para averiguar se, efetivamente, se podem identificar mudanças na dinâmica da comunidade quando recorremos ao mecanismo de gamificação de apoio (endorsement) de crachás. Para isso, foi escolhida a comunidade criada no âmbito do MOOC "Crachás: como usar?" que se iniciou a 20 de Abril de 2017 com atividades de ambientação à Plataforma SAPO Campus, procurando, também, que os membros criassem alguma afinidade entre si. Uma das atividades consistiu no apoio de crachás, em que os membros teriam de recomendar a atribuição de um dos crachás disponíveis perante a apresentação disponibilizada por cada colega. Neste artigo analisa-se o efeito desta atividade na dinâmica da comunidade.

\section{Plataforma SAPO Campus}

A plataforma SAPO Campus foi especialmente desenvolvida para utilização em contexto escolar (Santos, 2016). Deste modo, para além de outras funcionalidades disponibilizadas, dá especial ênfase às ferramentas de comunicação e inclui, também, uma ferramenta de criação e atribuição de crachás sem limite de utilização.

Nesta plataforma cada comunidade organiza-se num Espaço, onde o administrador pode gerir os acessos dos vários membros. Em cada Espaço é possível criar Grupos (subcomunidades) onde apenas membros desse Espaço podem participar. É importante referir que qualquer membro de um Espaço pode criar Grupos.

Apenas os administradores (Espaço/Grupo) têm a possibilidade de criar e atribuir crachás. Mas esta plataforma tem uma particularidade em relação a outras que disponibilizam crachás: permite que todos os membros possam apoiar a atribuição de crachás a outros membros que considerem merecedores desse símbolo. Esta funcionalidade informa o administrador de que um membro sugeriu que seja atribuído um crachá específico a um outro membro. Esta informação é, depois, associada ao próprio crachá e, caso o administrador decida atribuí-lo, o destinatário do crachá toma conhecimento de quem propôs/reconheceu que era merecedor daquele símbolo ( $\mathrm{Pe}$ dro, Santos, Aresta, \& Almeida, 2015).

\section{Contexto}

O MOOC "Crachás: como usar?"2 decorreu entre 18 de abril e 1 de junho de 2017, mas existiu um prolongamento das suas atividades até ao final do mês de junho. Este MOOC tinha como objetivo apresentar o conceito de crachá e discutir potenciais 
formas de utilização em contexto educativo ou de formação. Os seus destinatários eram professores e formadores de diferentes níveis de ensino ou faixas etárias.

As inscrições iniciaram-se duas semanas antes do início, tendo sido feita a divulgação em redes sociais e através da própria plataforma. Um total de 217 pessoas manifestaram-se interessadas, das quais apenas 131 efetivamente aderiram à comunidade online (Araújo, Santos, Pedro, \& Batista, 2017).

Durante a fase de ambientação e socialização em que decorreram as apresentações dos participantes no MOOC, foi proposta uma atividade em que os participantes eram convidados a apoiar crachás às apresentações feitas pelos colegas. Esta tarefa teve como objetivo incentivar a interação e a partilha dentro da comunidade criada e, também, possibilitar-Ihes uma experiência com crachás.

Importa descrever detalhadamente o progresso da comunidade. A partir de 18 de abril foi pedido a todos os inscritos, através de email, que aderissem ao Espaço Crachás: como usar? (Comunidade) para assim poderem ter acesso às atividades previstas no MOOC. Com o arranque oficial a 20 de abril, foi proposto que todos os participantes aderissem ao Grupo "Apresentações - $1^{\text {a }}$ semana" e realizassem a sua apresentação, podendo comentar as apresentações dos colegas. A 24 de abril foi, então, proposta a segunda atividade que solicitava a leitura das apresentações realizadas e a potencial manifestação de apoio aos autores dessas apresentações através de um dos crachás disponíveis (Figura 1). Inicialmente estavam previstos apenas cinco crachás, mas alguns membros solicitaram, entretanto, a criação de mais dois: Competitivo/a e Motivador(a)/Dinâmico(a).

Esta atividade prolongou-se por mais uma semana, até 4 de maio. Ao longo destas duas semanas os inscritos foram aderindo ao Espaço Crachás: como usar? e, posteriormente, ao Grupo onde iam realizando as atividades propostas.

O impacto que esta atividade teve na dinâmica da comunidade foi bastante revelador para os organizadores. Até ao momento em que foi lançada a segunda atividade, a 24 de abril, perto de metade dos membros já tinham realizado a sua apresentação e encontrávamos maioritariamente comentários do autor da publicação como resposta ao administrador que, para incentivar a interação, deixava sempre um comentário a cada nova publicação. Apenas 3 membros tinham realizado comentários em publicações que não eram da sua autoria nem do administrador. Após o lançamento desta atividade, as apresentações em falta começaram a surgir com mais frequência e, para além dos apoios, quarenta membros desencadearam comentários em diferentes apresentações de colegas.

Foi interessante verificar que, perante apresentações de novos membros, os que já estavam em tarefas posteriores regressavam a este grupo para as apoiar com crachás. 


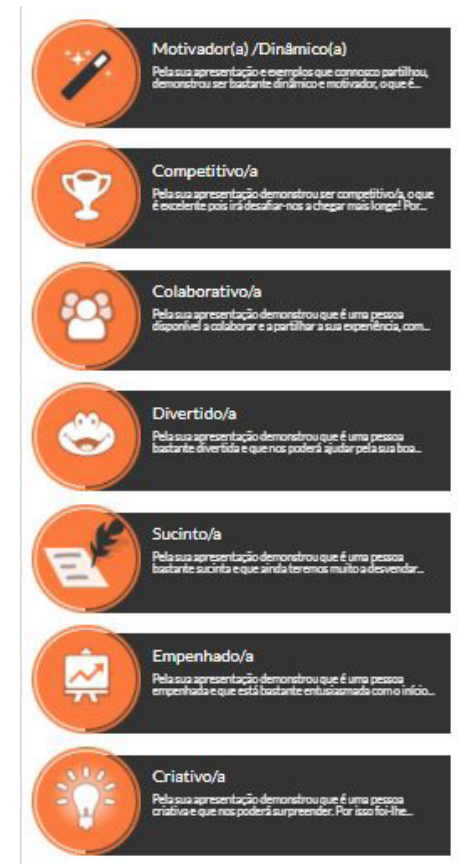

Figura 1 - Lista de crachás disponíveis para apoio em função da categorização das apresentações dos membros pertencentes ao grupo Apresentações - $1^{\text {a }}$ Semana

No final, ao avaliar a atividade da primeira semana, os membros apresentaram comentários como:

- "Considerei esta atividade divertida, democrática e de certo modo justa. (MS, 27 Abr, 14:44)"

- "Ainda ando meio aos "papéis" :-) mas já recebi 1 crachá... para começo, mto bom! (AE, 27 Abr, 17:19)"

- "Excelente e simpática entrada na matéria (:)! Para além de ter sido/ser um divertido quebra-gelo (NF, 28 Abr, 09:50)"

- "Gerou sensações que, a meu ver, são o motor da aprendizagem efetiva. Continuação de bom trabalho, que eu agora já tenho o meu primeiro crachá, que deixou tão motivada e orgulhosa! :) (SA, 28 Abr, 23:02)"

- "Adorei a forma divertida como se iniciou este MOOC (ES, 1 Mai, 13:25)"

Estes comentários são elucidativos sobre o impacto que as estratégias de apoio através de crachás poderão ter em comunidades online.

Para determinar se existiu algum impacto efetivo desta atividade na interação da comunidade recorreu-se a técnicas de SNA que apresentamos de seguida. 


\section{Metodologia}

A propósito de comunidades de aprendizagem online e, em particular, do surgimento de nós e ligações numa rede, Ferreira (2016, p. 126) afirma que

“(...) uma rede pode ser representada matematicamente por um grafo que descreve a sua estrutura topológica, sendo constituído por um conjunto de nós, também denominados por vértices, ligados por linhas que expressam a relação entre eles, também designadas por arestas. Usando um grafo para fazer a representação de uma rede social, os nós representam as pessoas e as arestas as relações entre elas".

Deste modo, e recorrendo a software específico, é possível representar graficamente as interações que ocorrem entre os membros de uma comunidade.

Um grafo "(...) consists of a set of objects, called nodes, with certain pairs of these objects connected by links called edges" (Easley \& Kleinberg, 2010, p. 23). Para a sua criação é necessário recolher dados que permitam identificar a fonte (source), o destinatário (target) e a interação ocorrida entre ambos (edge). É possível, ainda, associar características que permitam distinguir as interações como, por exemplo, a data em que a interação ocorreu ou um descritor sobre a interação ocorrida, facilitando a sua distinção (Cabral, 2016; Costa, 2013; Ferreira, 2016).

Através de técnicas de SNA é possível determinar medidas de centralidade como o valor médio de grau, que indica o número médio de membros com quem o autor de cada interação se relaciona (Ferreira, 2016). Outra medida é a densidade da comunidade, que corresponde à proporção do número de interações realizadas face ao número possível de realizar (Ferreira, 2016). Recorrendo a estas medidas e analisando a dispersão dos nós num grafo é possível perceber como a comunidade interagiu, se todos ou apenas alguns participaram nas interações e se houve formação de subgrupos.

É possível extrair dados de SNA a partir do motor de notificações geradas em cada Espaço do SAPO Campus (Cabral, 2016). Para isso recorreu-se ao software Gephi para aplicar métricas de SNA no tratamento dos dados que foram recolhidos sobre as interações no decurso do MOOC em estudo. Os dados usados na análise descrita neste artigo referem-se às duas primeiras semanas, intervalo em que ocorreram a maioria das interações relativas ao Grupo "Apresentações - $1^{\text {a }}$ semana", nomeadamente entre os dias 18 de abril e 4 de maio. Importa referir que a 27 de abril iniciaram-se as atividades relativas ao grupo "O que são crachás? - 2a semana", que não envolveram o apoio de crachás, mas apenas a realização de publicações ou comentários a publicações.

A análise dos dados extraídos permitiu verificar que há membros que pertencem a mais do que um Espaço (uma das características da plataforma SAPO Campus) e, por 
isso, surgem interações com membros desses outros Espaços. Como esses outros espaços não faziam parte deste estudo, foi então necessário proceder à verificação dos nomes identificados nos nós, tendo sido eliminados todos os nós (nodes) e respetivas linhas (edges) que envolviam nomes, quer na fonte quer no destinatário, que não pertenciam ao Espaço Crachás: como usar?

A extração de dados pelo SAPO Campus permite identificar o tipo de relação de cada linha (edge). Na Tabela 1 apresentam-se os códigos e os respetivos significados, tendo em conta o funcionamento da plataforma.

\begin{tabular}{|c|c|c|}
\hline \multicolumn{2}{|c|}{ Código } & Significado \\
\hline & $\begin{array}{l}\text { GroupPublish } \\
\quad(56,3 \%)\end{array}$ & $\begin{array}{l}\text { Publicações realizadas no grupo com uma tipologia de interação de um } \\
\text { para todos os membros. }\end{array}$ \\
\hline & $\begin{array}{l}\text { Badge_Suport- } \\
\text { Pending } \\
(10,8 \%)\end{array}$ & $\begin{array}{l}\text { Apoio de crachá a um membro, com uma tipologia de interação do tipo } \\
\text { um para um. }\end{array}$ \\
\hline & $\begin{array}{l}\text { Highlight } \\
(10,3 \%)\end{array}$ & $\begin{array}{l}\text { Destaque de publicação notificando os membros por correio eletrónico, } \\
\text { com uma tipologia de interação do tipo um para todos. }\end{array}$ \\
\hline & $\begin{array}{l}\text { Comment Also } \\
(7,0 \%)\end{array}$ & $\begin{array}{l}\text { Comentário realizado numa publicação em que não é autor, interação } \\
\text { de um para o autor e outros membros que também interagiram com a } \\
\text { publicação. }\end{array}$ \\
\hline & $\begin{array}{l}\text { Comment Author } \\
\qquad(4,1 \%)\end{array}$ & $\begin{array}{l}\text { Comentário realizado numa publicação em que é autor, interação de um } \\
\text { para o autor da publicação. }\end{array}$ \\
\hline & $\begin{array}{l}\text { Follow } \\
(3,7 \%)\end{array}$ & Seguir pessoa, interação do tipo um para um (pessoa). \\
\hline & $\begin{array}{l}\text { GroupJoin } \\
\quad(1,7 \%)\end{array}$ & Aderir a um grupo, interação de um para administrador(es) do grupo. \\
\hline & $\begin{array}{l}\text { ArticleAdd } \\
\quad(1,4 \%)\end{array}$ & $\begin{array}{l}\text { Publicação de artigo no blogue, interação do tipo um para vários } \\
\text { (seguidores). }\end{array}$ \\
\hline & $\begin{array}{l}\text { GroupSelfinvite } \\
(0,9 \%)\end{array}$ & Pedido de adesão ao grupo, interação de um para administrador(es). \\
\hline & $\begin{array}{l}\text { Favorite } \\
(0,9 \%)\end{array}$ & $\begin{array}{l}\text { Marcar como favorita uma publicação, interação de um para autor da } \\
\text { publicação. }\end{array}$ \\
\hline & $\begin{array}{l}\text { GroupAccept } \\
(0,9 \%)\end{array}$ & Aceitação de adesão ao grupo, interação do tipo um para um. \\
\hline & $\begin{array}{l}\text { ServiceSubscribe } \\
\quad(0,8 \%)\end{array}$ & Seguir blogue, interação do tipo um para um ou vários (autores do blog). \\
\hline & $\begin{array}{l}\text { Badge_suport } \\
\quad(0,7 \%)\end{array}$ & Novo apoio a crachá já atribuído, interação do tipo um para um. \\
\hline & $\begin{array}{l}\text { PersonTag } \\
(0,6 \%)\end{array}$ & $\begin{array}{l}\text { Mencionar membro em publicação ou comentário, interação do tipo um } \\
\text { para um. }\end{array}$ \\
\hline
\end{tabular}

Tabela 1 - Descrição da tipologia de interação em cada linha (Edge) com a cor com que é representada nos Grafos (ver Tabela 2) e \% de interações realizadas de cada tipo. 
Importa realçar que há relações que geram interações de um membro para todos os outros que pertencem ao Grupo/Espaço, como é o caso das publicações e dos destaques a publicações. Há outras relações que geram interações do tipo um para alguns membros, como é o caso dos comentários onde apenas os autores das publicações e outros membros que tenham igualmente comentado recebem a notificação. Há também relações do tipo um para um, como é o caso de quem segue outro membro ou mesmo o apoio de crachás.

O facto de mais de metade das interações serem publicações no Grupo/Espaço é de certo modo expectável, pois cada uma dessas interações gerou o mesmo número de linhas que o número de elementos existentes nesse Grupo/Espaço. Ou seja, sempre que há uma publicação são geradas notificações individuais a partir do autor para cada um dos outros membros, por isso é fácil que este tipo de relação gere inúmeras linhas (edges).

Um facto a realçar é que o Apoio de crachás a membros, que é uma interação do tipo um para um, gerou o segundo maior número de interações, tendo sido identificadas 931 linhas (edges). Isto revela um grande esforço realizado pelos membros neste tipo de interação.

A seguir apresenta-se a análise dos grafos extraídos da ferramenta de SNA e a sua evolução ao longo de vários dias.

\section{Discussão dos dados}

Ao todo foram identificados 131 nós (nodes) diferentes, sendo que, destes, apenas 98 tinham desencadeado interações (source). Foi também identificado um total de 8654 linhas de interação (edge).

Recorrendo à aplicação Gephi 0.9.2 foi analisado um ficheiro .csv contendo as seguintes colunas: target, source, date, relationship_type, seguindo procedimentos realizados em estudos anteriores com a plataforma SAPO Campus (Cabral, 2016; Ferreira, 2016; Pedro et al., 2016). Procedeu-se então à criação do respetivo grafo utilizando o algoritmo predefinido Force Atlas 2, sendo que todas as linhas de interação possuem o valor de peso (weight) igual, ou seja, neste caso, 1. Para averiguar o impacto que a atividade de Apoio de crachás exerceu na dinâmica da comunidade procedeu-se à recolha de imagens dos Grafos relativas às interações diárias entre os dias de 18 de abril a 4 de maio (Tabela 2) e também à recolha de métricas de centralidade (Figuras 3 e 4). 
Dia e atividades promovidas pelo administrador

18 Abril

Email enviado a convidar os inscritos a aderir ao Espaço.

19 Abril

Publicação de boas vindas no Espaço.

\section{Abril}

Publicação de arranque da formação no Espaço e respetivo destaque.

Publicação de Tarefa e destaque da publicação.

\section{1 abril}

Publicação de informação no Espaço sobre o início das atividades no grupo da $1^{\mathrm{a}}$ semana e respetivo destaque.
Grafo
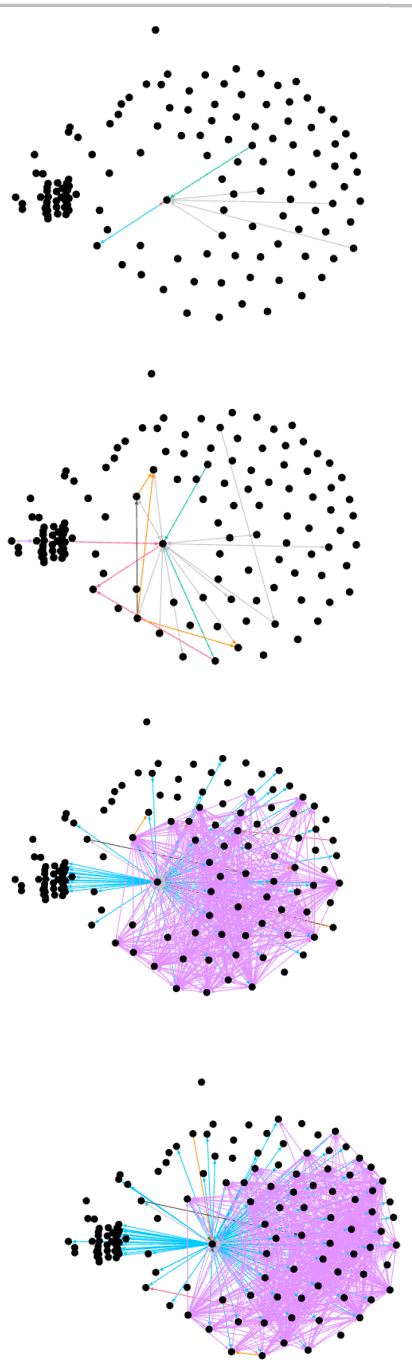
Dia e atividades promovidas pelo administrador

\section{2 abril}

Sem ações significativas por parte do administrador.

\section{3 abril}

Publicação com orientação sobre como marcar uma tarefa como concluída no grupo e respetivo destaque.

Atribuição de Crachá ao $100^{\circ}$ membro e publicação do seu anúncio.

\section{4 abril}

São dadas instruções para a tarefa de apoiar a crachás às apresentações de membros.

\section{5 abril}

Destaque da publicação da Tarefa de apoiar a crachás às apresentações de membros.

\section{6 abril}

Sem ações significativas por parte do administrador.

\section{Grafo}
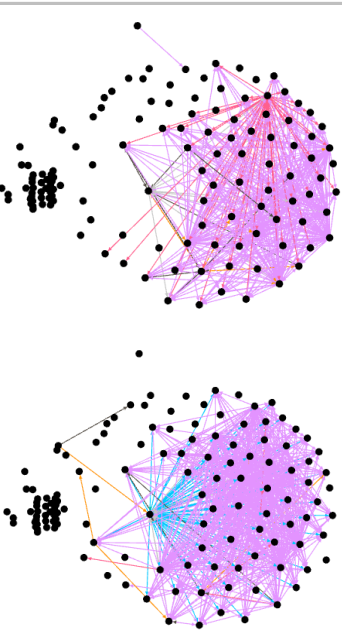

•
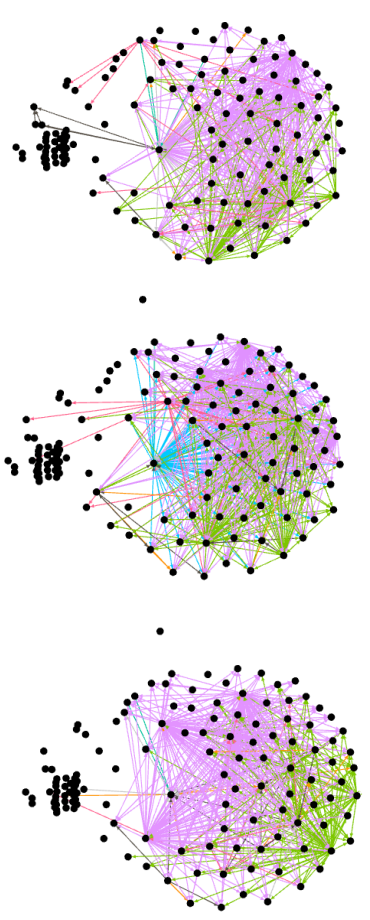
Dia e atividades promovidas pelo administrador

27 abril

Publicação e destaque do anúncio de atribuição de Crachás no Grupo.

Atribuição de 39 crachás.

Publicação e destaque no Espaço do arranque do grupo da $2^{a}$ semana.

28 abril

Atribuição de 14 crachás.

Publicação e destaque de artigo em blog dentro do grupo da $2^{a}$ semana.

\section{9 abril}

Sem ações significativas por parte do administrador.

\section{0 abril}

Sem ações significativas por parte do administrador.

\section{1 maio}

Publicação para apoiarem crachás a quem ainda não recebeu.

\section{Grafo}
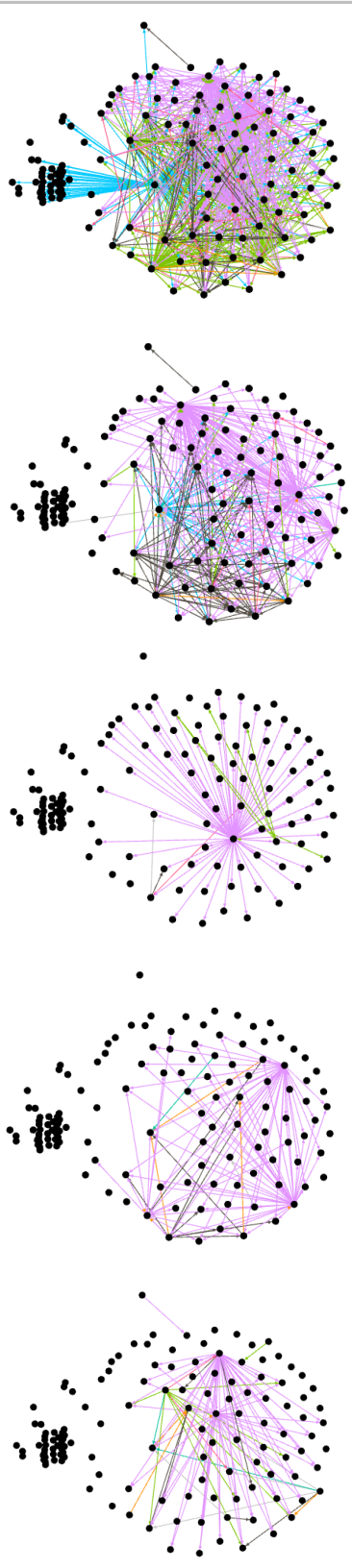
Dia e atividades promovidas pelo administrador

\section{2 maio}

Publicação e Destaque no grupo da $1^{\text {a }}$ semana pedindo para aderirem ao grupo da $2^{\mathrm{a}}$ semana assim que finalizem as atividades.

Publicação e destaque de artigo em blogue no grupo da $2^{a}$ semana.

\section{3 maio}

Publicação e destaque de tarefa a realizar no grupo da $2^{a}$ semana.

\section{4 maio}

Publicação e destaque de mensagem a solicitar a adesão aos grupos por nível de ensino.
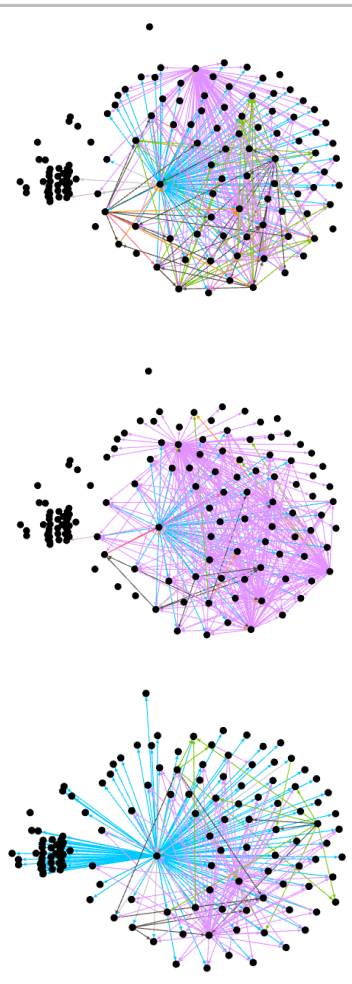

Tabela 2 - Descrição das atividades realizadas pelo administrador do Espaço/Grupo em cada um dos dias no período de 18 de abril a 4 de maio com os respetivos grafos de interações geradas (ver legenda do tipo de interação codificada na Tabela 1).

Uma primeira análise da distribuição dos nós (edges) nos grafos permite identificar que existe um grupo no lado esquerdo e que se encontra na orla exterior da circunferência formada pelos nós que mais participam nas interações. Estes nós à esquerda representam os membros que pertencem ao Espaço, mas que não chegaram a entrar no Grupo onde decorreram as atividades. São principalmente recetores de interações, meros observadores, este tipo de participação (lurking) é muito usual nas comunidades online (Salmon, 2003; Wenger, 2011). Existe também um nó que se destaca, pois dele surgem sempre as publicações de destaque (azul claro). Este nó corresponde ao administrador do Espaço e do Grupo, responsável por dinamizar as tarefas do MOOC. É importante referir que este ponto não se encontra ao centro da circunferência, algo 
que seria expectável caso fosse o membro que desencadeasse e recebesse a maioria das interações. É interessante observar que a maioria dos nós estão dispersos numa circunferência quase perfeita, sendo que se aproximam do centro os que mais vezes iniciaram e receberam interações.

Analisando os 16 Grafos ao longo da Tabela 2 é possível identificar mudanças que resultam das diferentes atividades. Os primeiros dias revelam uma evolução gradual em termos de publicações no Espaço/Grupo. Verificaram-se alguns apoios de crachás logo desde o dia 20 de Abril, mas são valores residuais. Isto ocorre, provavelmente, devido à curiosidade de alguns participantes e pelo facto de os Crachás serem o próprio objeto de estudo na comunidade. Mas é no dia 24 de abril, no dia em que são dadas instruções aos participantes para apoiarem crachás associados às apresentações, que este tipo de interação aumenta entre os membros, criando uma rede densa nos grafos dos dias seguintes. É de realçar que no dia 25 de Abril, com o destaque e subsequente envio por email da informação sobre a tarefa de apoio de crachás, ocorre o valor máximo de apoios, 265 (ver figura 2). É também notável a diversidade de tipos de relações, sendo os comentários a publicações uma das que se destaca nos grafos dos dias 27 e 28 de abril.

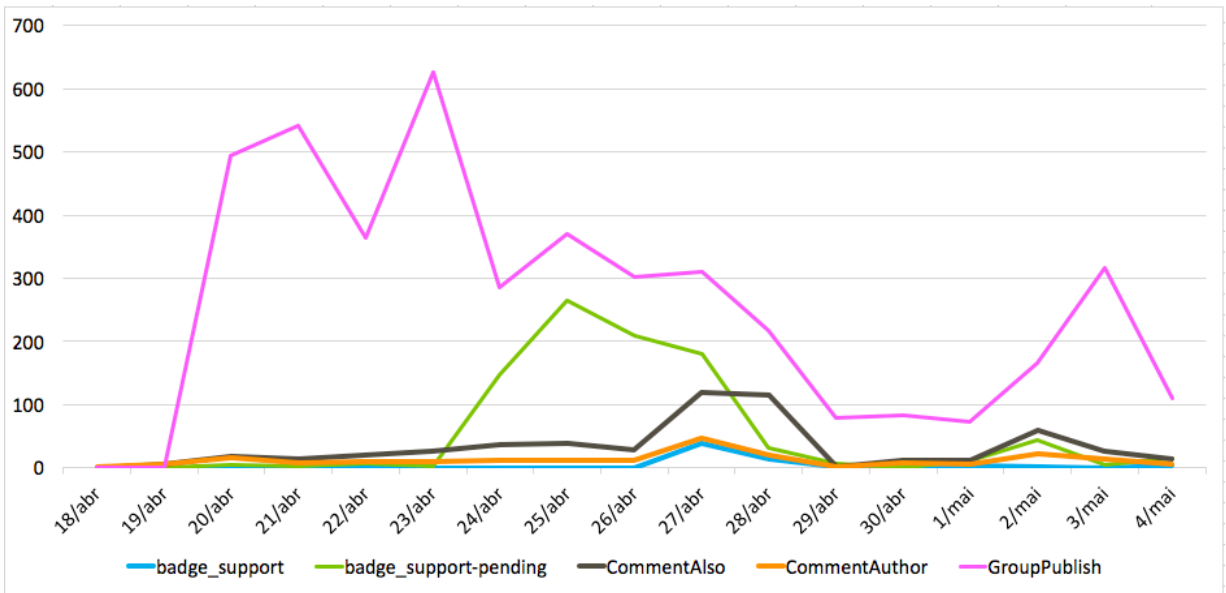

Figura 2 - Oscilação do número de interações (Crachás, Comentários e Publicações) desencadeadas apenas pelos membros entre os dias 18 de abril e 4 de maio $(n=5939)$.

Pelo gráfico na figura 2 é possível analisar a quantidade de interações realizadas apenas pelos membros, significa isto que interações que tinham o administrador como autor (source) foram retiradas. Este gráfico tem o intuito de compreender o contributo dos membros para as interações dentro da comunidade ao nível das Publicações, Crachás e Comentários. Verificamos que existe um máximo de 627 interações do tipo GroupPublish no dia 23 de abril (domingo), dia em que o alerta para concluir a tarefa foi ativado 
e o administrador informou como é que os participantes deviam proceder. Somando todas as interações percebemos que nos dias 25 de abril (feriado) e o dia 27 de abril as interações atingem novos máximos, de 686 e 697 respetivamente. Significa isto que as interações GroupPublish (tipo um para todos) foram parcialmente substituídas por interações do tipo um para um (Crachás) e um para vários (Comentários) o que revela um maior esforço por parte dos membros em comparação com os primeiros dias.

Estes dados revelam um maior esforço por parte dos membros no período de 24 a 28 de abril, tendo o ritmo de publicações no Grupo diminuído e aumentado os debates através de comentários. Esta mudança foi posterior à proposta da tarefa de apoio de crachás.

Pela análise de figura 3 podemos verificar que o grau médio dos participantes da comunidade apresenta um máximo de 9,02 no dia 27 de abril. O mesmo se verifica quando é analisada a densidade da comunidade, que atinge um máximo de 0,069 no dia 27 de abril (Figura 4). Comparando com a figura 2 verificamos que o dia 27 de abril, onde são atingidos estes máximos, é também o dia em que há maior diversidade de interações, facto também visível na combinação de ligações de diferentes cores que o Grafo de dia 27 de abril apresenta (Tabela 2).

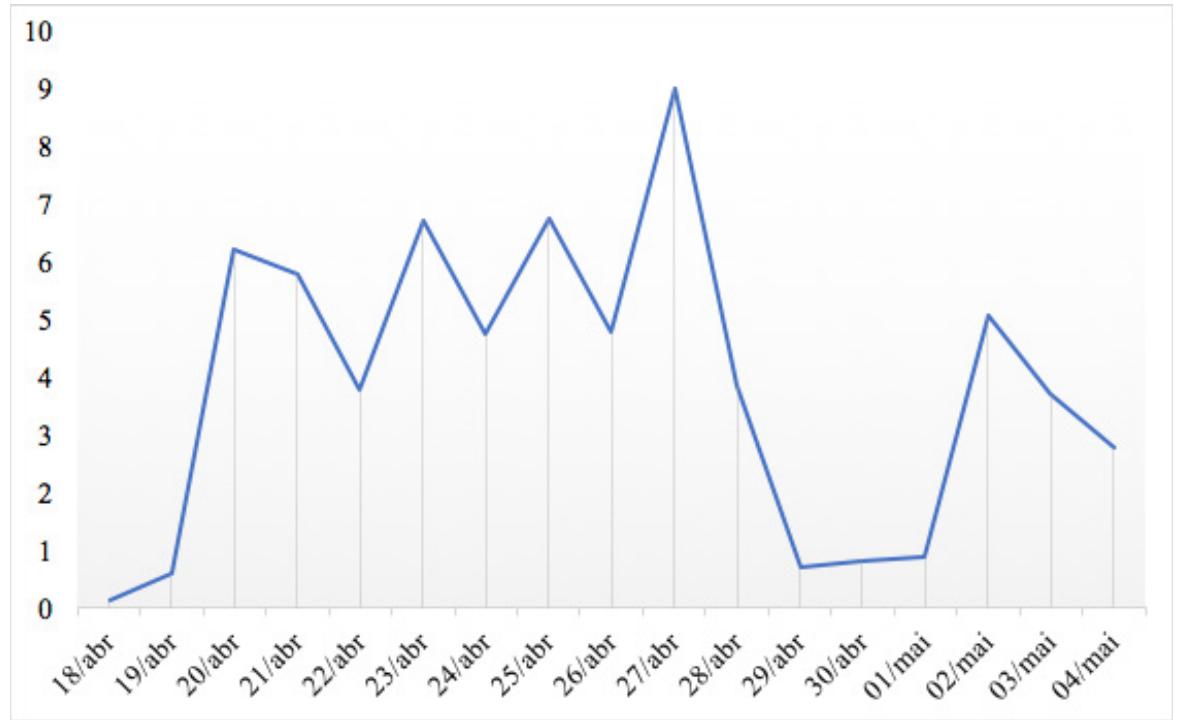

Figura 3 - Oscilação do valor médio de Grau dos participantes da Comunidade Crachás entre os dias 18 de abril e 4 de maio. 


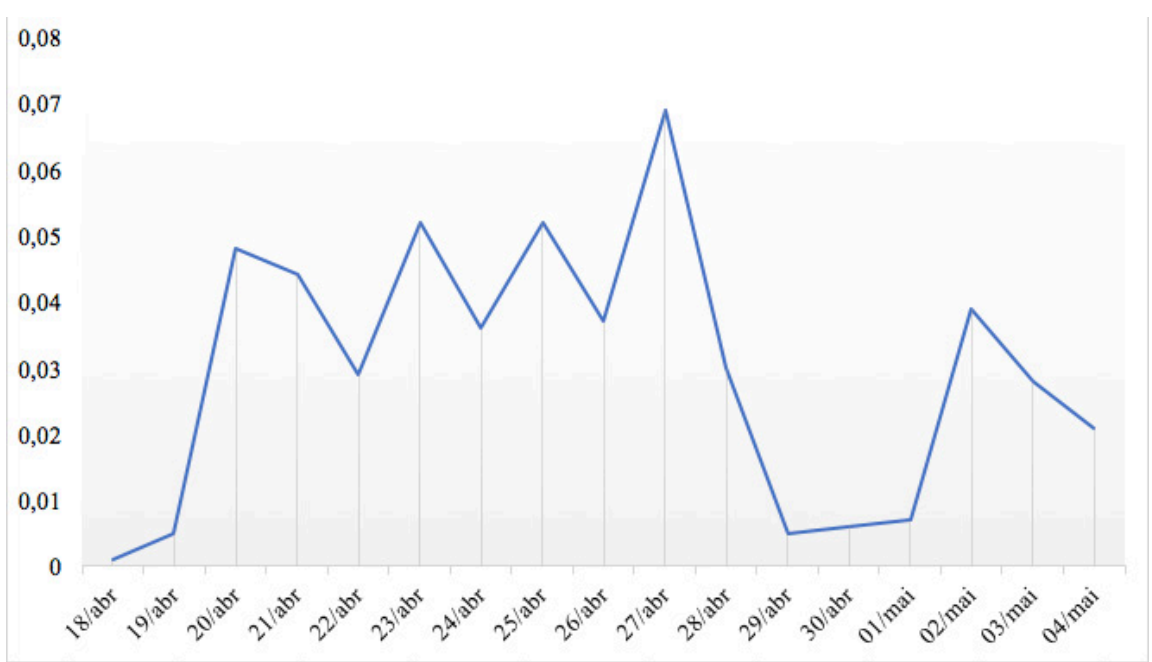

Figura 4 - Oscilação do valor de Densidade da Comunidade Crachás entre os dias 18 de abril e 4 de maio.

Podemos também associar esses máximos no dia 27 de abril ao facto de ter sido nesse dia que foram atribuídos os primeiros 39 crachás, o que levou muitos membros a entrarem na comunidade e a participar.

Apesar de estes dados serem reveladores de que algo, efetivamente, provocou uma maior densidade de interações, não podemos concluir que os únicos responsáveis são os crachás. É fácil identificar que nos dias em que uma publicação é destacada, os valores apresentam também aumentos.

A fase de ambientação num MOOC é sempre propícia a que, quem esteja movido pela curiosidade, adira à comunidade e participe nela de forma ativa. Ou mesmo para outros membros que apenas desejam conhecer como foi estruturado esse novo curso, para assim implementar técnicas semelhantes em projetos próprios (Onah, Sinclair, \& Boyatt, 2014). É por isso uma fase em que, habitualmente, as interações e participação nas tarefas são mais frequentes.

No entanto, através da observação dos comentários realizados pelos participantes durante a avaliação no final da atividade (ver acima), verifica-se que a possibilidade de apoiarem crachás e a curiosidade de saberem que crachá iriam receber criou um momento divertido, permitindo alguma desinibição. Este facto está em linha com outro estudo em que se afirma que "students (...) said that the Badges made them more visible in the Network and help them learn with each other" (Bacelar, Morgado, \& Rocio, 2017, p. 3). De facto, foi a partir desse momento que outras formas de interação, como o comentário a publicações, ganharam maior evidência. 
Importa ainda realçar que o facto de o dia 25 de abril ser um feriado nacional, não impediu que tivesse sido o dia em que ocorreram mais interações de apoio de crachás $(n=265)$. Por outro lado, no dia 1 de maio, igualmente um feriado nacional, e que neste caso ocorreu numa segunda-feira prolongando o fim de semana, verificou-se uma desaceleração de todas as atividades na comunidade.

\section{Considerações Finais}

Consideramos estes dados reveladores, mas também reconhecemos que é necessária uma avaliação mais profunda. É necessário recorrer a outras experiências para perceber se os crachás poderão ser uma ferramenta que, efetivamente, envolve os formandos em comunidades online. É necessário também comparar os efeitos que apenas a atribuição de crachás poderá provocar face ao ato de apoiar crachás.

Surge, assim, uma nova potencialidade de utilização dos crachás em comunidades online, que necessita ser investigada e avaliada com maior profundidade.

\section{Agradecimentos}

Este trabalho é financiado por fundos nacionais através da FCT - Fundação para a Ciência e a Tecnologia, I.P., no âmbito do projeto GamiLearning, Jogos Digitais para a Literacia Mediática e Informacional (UTAP-ICDT/IVC-ESCT/0020/2014)

\section{Notas}

Link: http://campus.sapo.pt/

Link: http://crachascomousar.campus.sapo.pt/

\section{Referências}

Araújo, I., Santos, C., Pedro, L., \& Batista, J. (2017). Crachás: Como usar? - Um MOOC na formação de professores. In 19th International Symposium on Computers in Education (SIIE). Lisboa: Escola Superior de Educação de Lisboa IPL.

Bacelar, F., Morgado, L., \& Rocio, V. (2017). Gamification Badges and Ratings: Impact on an Academic Social Network. In 1st Workshop on Gamification and Games for Learning (GamiLearn'17). Puerto de la Cruz (Tenerife): Universidad de La Laguna. https://riull.ull.es/xmlui/handle/915/4782

Costa, M. da C. G. (2013). Marcas, literacia mediática e expressões de identidade dos préadolescentes. Tese de doutoramento. Lisboa: Universidade Nova de Lisboa. https://run.unl.pt/ handle/10362/10405

Cabral, G. V. (2016). Exportação de dados para análise de redes sociais: implementação e avaliação de uma ferramenta. Dissertação de Mestrado. Aveiro: Universidade de Aveiro. http://ria.ua.pt/ handle/10773/17802 
De Lima, M., \& Zorrilla, M. E. (2017). Social Networks and the Building of Learning Communities: An Experimental Study of a Social MOOC. The International Review of Research in Open and Distributed Learning, 18(1). https://doi.org/10.19173/irrodl.v18i1.2630

Easley, D., \& Kleinberg, J. (2010). Networks, crowds, and markets: Reasoning about a highly connected world. Cambridge: Cambridge University Press.

Ferreira, M. de F. G. P. (2016). SAPO campus: aprendizagem, ensino e pessoas em rede. Tese de doutoramento. Aveiro: Universidade de Aveiro. http://ria.ua.pt/handle/10773/15855

Gee, J. P. (2003). What Video Games have to teach us about learning and literacy. EUA: Palgrave Macmillan.

Hamari, J. (2017). Do badges increase user activity? A field experiment on the effects of gamification. Computers in Human Behavior, 71, 469-478. https://doi.org/10.1016/j.chb.2015.03.036

Looyestyn, J., Kernot, J., Boshoff, K., Ryan, J., Edney, S., \& Maher, C. (2017). Does gamification increase engagement with online programs? A systematic review. PLOS ONE, 12(3), https://doi. org/10.1371/journal.pone.0173403

Onah, D. F. O., Sinclair, J., \& Boyatt, R. (2014). Dropout rates of massive open online courses : behavioural patterns. In 6th International Conference on Education and New Learning Technologies (pp. 5825-5834). Barcelona, Spain: EDULEARN. http://wrap.warwick.ac.uk/65543/

Pedro, L., Santos, C., Aresta, M., \& Almeida, S. (2015). Peer-supported badge attribution in a collaborative learning platform: The SAPO Campus case. Computers in Human Behavior, 51, 562-567. https:// doi.org/10.1016/j.chb.2015.03.024

Pedro, L., Santos, C., Batista, J., Cabral, G., Pais, F., \& Costa, C. (2016). Social Network Analysis and Digital Learning Environments: a Framework for Research and Practice Using the Sapo Campus Platform. In 10th International Technology, Education and Development Conference (INTED2016) (pp. 1061-1070). Valencia, Spain: IATED. https://doi.org/10.21125/inted.2016.1239

Rebollo-Catalán, M. A., García-Pérez, R., Buzón-García, O., \& Vega-Caro, L. (2014). Las emociones en el aprendizaje universitario apoyado en entornos virtuales: diferencias según actividad de aprendizaje y motivación del alumnado. Revista Complutense de Educación, 25(1), 69-93. https:// doi.org/10.5209/rev_RCED.2014.v25.n1.41058

Salmon, G. (2003). E-moderating: The Key to Teaching and Learning Online (Second Edi). London: RoutledgeFalmer.

Santos, C. M. das N. (2016). Estudo, concepção e desenvolvimento de uma plataforma integrada de serviços web 2.0 para utilização em contexto de ensino superior. Tese de doutoramento. Aveiro: Universidade de Aveiro. http://ria.ua.pt/handle/10773/18603

Wenger, E. (2011). Levels of participation - Multiple ways to engage in social learning. (Disponível em https://wenger-trayner.com/project/levels-of-participation/ consultado em 15/12/2017). 
Inês Araújo

Bolseira de Investigação no âmbito do projeto GamiLearning (http:// gamilearning.ulusofona.pt/pt/) Universidade de Aveiro, Aveiro, Portugal

Email: inesaraujo@ua.pt / inesjgc@gmail.com ORCID: https://orcid.org/0000-0003-0936-1411

Carlos Santos

DigiMedia, DECA, Universidade de Aveiro, Aveiro, Portugal

Email: carlossantos@ua.pt ORCID: https://orcid.org/0000-0003-0961-8088

Luís Pedro

DigiMedia, DECA, Universidade de Aveiro, Aveiro, Portugal

Email: Ipedro@ua.pt ORCID: https://orcid.org/0000-0003-1763-8433

João Batista

DigiMedia, ISCA, Universidade de Aveiro, Aveiro, Portugal

Email: joao.batista@ua.pt

ORCID: http://orcid.org/0000-0002-5872-5341

Correspondência:

Departamento de Comunicação e Arte

Campus Universitário de Santiago

Universidade de Aveiro

3810-193 Aveiro

Data de submissão: Janeiro 2018 Data de avaliação: Março 2018

Data de publicação: Julho 2018 